\title{
Medición distribuida de la calidad de la energía eléctrica
}

\section{Distributed measurement of power quality}

DOI: $10.46932 / \mathrm{sfjdv} 3 \mathrm{n} 1-079$

Received in: Jan 30st, 2021

Accepted in: Feb 1th, 2022

\section{Armando Mora Campos}

Profesor de Ingeniería Electrónica en el Tecnológico Nacional de México campus Querétaro

E-mail: armando.mc@queretaro.tecnm.mx

\section{Jöns Sánchez Aguilar}

Profesor de Ingeniería Industrial y de Ciencias Básicas en el Tecnológico Nacional de México campus

Querétaro

E-mail: jonssanchez@gmail.com

\section{Hernando Chagolla Gaona}

Profesor de Ingeniería Electrónica y Mecatrónica en el Tecnológico Nacional de México campus Querétaro

E-mail: hchagollag@ hotmail.com

\section{Carlos Colín Hernández}

Estudiante del onceavo semestre de Ingeniería Electrónica en el Tecnológico Nacional de México campus Querétaro

E-mail: carloscolinhernandez@outlook.com

\section{RESUMEN}

Se presenta la primera etapa de una aplicación de Internet de las Cosas en los sistemas eléctricos, documentando el diseño de un nodo final para la medición de la calidad de la energía en redes trifásicas y el protocolo de red de bajo consumo y largo alcance (LoRaWAN) en el que este nodo se activa. El objetivo es desarrollar un equipo de medición para la realización de auditorías de calidad de la energía eléctrica en pequeñas y medianas empresas. Como resultados se tienen la especificación de la red a utilizar y la tarjeta electrónica del nodo final con la funcionalidad deseada. Esta aplicación busca aprovechar la capacidad de Internet de las Cosas en el desarrollo de sistemas de medición distribuida con altos niveles de seguridad y confiabilidad.

Palabras clave: IoT, LoRaWAN, energía eléctrica.

\footnotetext{
ABSTRACT

The first stage of an Internet of Things application in power systems is presented, documenting the design of an end node for power quality measurement in three-phase networks and the low-power, long-range network (LoRaWAN) protocol in which this node is activated. The objective is to develop a measurement equipment for performing power quality audits in small and medium-sized companies. The results are the specification of the network to be used and the electronic card of the final node with the desired
} 
functionality. This application seeks to take advantage of the Internet of Things capability in the development of distributed metering systems with high levels of security and reliability.

Keywords: IoT, LoRaWAN, electric power.

\section{INTRODUCCIÓN}

De acuerdo a estudios recientes (Chandrappa y Banerjee, 2017), los problemas en la energía eléctrica provocan entre otras consecuencias, mal funcionamiento, calentamiento y daño a los aislamientos de los equipos industriales, causando pérdidas económicas debido a paros de producción inesperados, alto costo de mantenimiento y envejecimiento prematuro de la maquinaria. La fuente principal de estos inconvenientes es el efecto de las cargas conectadas en la red propia, y que además se propaga o se recibe de edificios y empresas adyacentes.

Los desórdenes energéticos se refieren técnicamente a problemas de calidad de la energía eléctrica. Consisten en variaciones de la alimentación debido básicamente a cuestiones de cableado, efectos de sistemas de tierras inadecuados, transitorios de conmutación, variaciones de carga y generación de armónicas. Chandrappa y Banerjee documentan que en Estados Unidos, las fuentes principales de los problemas de calidad de energía son caídas de tensión (48\%), armónicas (22\%), cableado y tierras (15\%), conmutación de capacitores (6\%), interacción de cargas (5\%), EMF/EMI (1\%), acondicionamiento de energía $(1 \%)$ y otros $(2 \%)$.

Para superar estas condiciones, el primer paso es monitorear las entradas de alimentación y los disturbios causados por la carga eléctrica, identificando las técnicas de mitigación que proporcionen al equipo industrial una energía limpia.

Una alternativa es la instalación de una red de monitores de calidad de la energía, para identificar fuentes de disturbios en diferentes secciones y circuitos simultáneamente. Los monitores se pueden definir como nodos finales de una red de Internet de las Cosas (en inglés Internet of Things, abreviado IoT), que transmiten datos a la nube, donde se analizan para identificar las perturbaciones que causan baja calidad de la energía. Este método de medición presenta la ventaja de tener varios puntos de observación y análisis localizados a través de la red eléctrica por largos periodos de tiempo (Antonesei, 2011). A continuación se hace referencia a la tecnología de IoT propuesta, así como al diseño del nodo de IoT con la funcionalidad solicitada. 


\section{RED LORAWAN}

\subsection{REDES DE INTERNET DE LAS COSAS}

El IoT hace referencia a la interconexión de objetos físicos a Internet, mediante sistemas electrónicos y tecnologías de la información y comunicación, con el propósito de integrar personas, procesos y sistemas, a partir de la extracción y gestión de cantidades masivas de datos del mundo real. Esto lo ha convertido en un símbolo de modernidad y un detonador del progreso social, comercial e industrial, motivando la innovación, el emprendimiento y la creatividad. En el desarrollo de redes de IoT, se han utilizado con éxito estándares y sistemas de comunicación tradicionales, como WiFi, Bluetooth, ZigBee, Z-Wave y telefonía celular 2G y 3G, en aplicaciones de domótica, wereables, control industrial, Smart City, Smart Grid, etc. (Components, 2015).

Para aplicaciones de alta cobertura y bajo consumo de energía, con necesidades de confiabilidad, seguridad, escalabilidad y penetración de la señal en edificios, se tienen estándares y protocolos de comunicación específicos para IoT. Entre ellos se encuentran el LTE Cat M1 y el LTE Cat NB1 para telefonía celular, el 802.11ah para Wi-Fi y el grupo de protocolos para redes WAN de baja potencia (LPWAN).

Las LPWAN son tecnologías de IoT utilizadas en aplicaciones donde se tienen requerimientos como gran alcance y bajo consumo de energía, usando una infraestructura simple y robusta (Quinnell, 2015). En este segmento emergente, varias empresas y alianzas buscan dominar el mercado con tecnologías y estándares propios, como SixFox, Ingenu RPMA, Weightless, Dash 7 y LoRaWAN.

\subsection{RED LORAWAN}

Dentro de las redes LPWAN, destaca una especificación de red, para interconectar dispositivos inalámbricamente en la banda industrial, científica y médica (ISM), alimentados por batería y con alcance de varios kilómetros, la red WAN de largo alcance y bajo consumo nombrada LoRaWAN (LoRa, 2018).

LoRaWAN es el nombre de una alianza y un protocolo de estándar abierto, que certifica y promueve los productos y servicios de sus más de 500 miembros. Además de la alta calidad tecnológica de su estándar, esta alianza se distingue por su apertura, al permitir que sus asociados adopten el modelo de negocios que más les convenga, lo que ha propiciado la existencia de redes LoRaWAN públicas y privadas en 144 países. Su arquitectura consiste de una topología estrella, que usa gateways como puentes de enlace transparentes entre los nodos finales y los servidores de gateways, red y aplicaciones (figura 1). 
Figura 1. Ejemplo de arquitectura de red LoRAWAN.

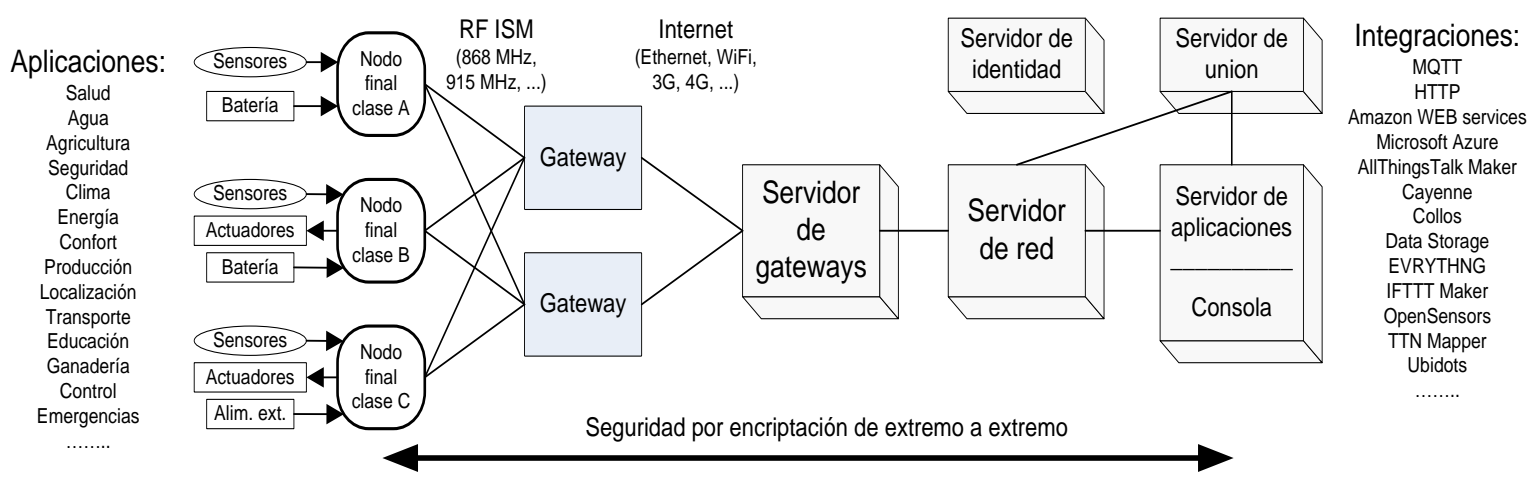

De acuerdo a la especificación LoRaWAN 1.1 (LoRaWAN, 2017), la comunicación de los nodos finales es generalmente bidireccional, pero también soporta operación multicast. La comunicación entre nodos y gateways se distribuye entre diferentes frecuencias y tasa de datos. La selección de la tasa de datos, en un rango de $0.3 \mathrm{kbps}$ a $50 \mathrm{kbps}$, se equilibra entre el alcance de la comunicación y la duración del mensaje. En base a su tecnología de espectro disperso, las comunicaciones con diferente tasa de datos no interfieren entre sí, creando un conjunto de canales virtuales que incrementan la capacidad del gateway. Para maximizar la vida de la batería de los nodos y la capacidad de la red, el servidor de red administra la tasa de datos y la salida de RF de cada nodo, por medio de un esquema adaptivo.

La especificación LoRaWAN satisface las necesidades de comunicación segura, utilizando diversas capas de encriptado: llave de red única (EUI64) y comunicación segura al nivel de red; llave de aplicación única (EUI64), para la seguridad extremo a extremo, al nivel de aplicación y llave de dispositivo específico (EUI128). También se incluyen tres clases de dispositivos de nodos finales, para dar solución a los requerimientos de las aplicaciones: clase A: nodos bidireccionales con dos ranuras fijas de recepción después de cada transmisión, para sensores alimentados con batería; clase B: nodos bidireccionales con ranuras de recepción programadas, para sensores y actuadores alimentados por batería; clase C: nodos bidireccionales con ranuras de recepción continuas, para sensores y actuadores alimentados por la energía principal.

\subsection{COMUNIDAD THE THINGS NETWORK}

Uno de los miembros de la alianza LoRa es la fundación holandesa The Things Network (TTN, https://www.thethingsnetwork.org/). La TTN es dirigida por un equipo de 16 miembros, incluyendo al iniciador Wienke Giezeman y al líder tecnológico Johan Stokking. Su lema, Building a global internet of things network together, refleja la iniciativa de construir redes de IoT propias, operadas por los usuarios. El interés del grupo es que más personas contribuyan instalando gateways, desarrollando software, formando comunidades (grupos organizados que usan su plataforma) o trabajando en aplicaciones. 
Para cerrar el ciclo de conectividad, TTN proporciona sin costo el apoyo de servidores en la nube y el uso del software de manejo de aplicaciones. Actualmente tiene presencia en 90 países, con más de 49500 miembros, 400 comunidades y 4580 gateways. La fundación TTN está por lanzar la tercera generación de su software de red (stack), el cual ofrecerá mayor seguridad, escalabilidad, velocidad y facilidad para el desarrollador. Con esto se busca cumplir con la premisa de que el futuro son las redes privadas conectadas.

\subsection{HARDWARE Y SOPORTE DE RED}

El hardware necesario para acceder a una red TTN son los nodos finales y los gateways. Los nodos comunican a la red los sensores y actuadores de una aplicación, utilizando microcontroladores de bajo consumo de energía y circuitos transceptores LoRa. La TTN ha desarrollado una interfaz para que los usuarios registren sus aplicaciones y gateways (https://console.thethingsnetwork.org/). Cada nodo se da de alta bajo una aplicación, en una de las dos modalidades de conexión, en forma dinámica con OTAA (On The Air Activation) o manualmente con ABP (Activation By Personalization).

Para tener funciones extras de almacenamiento, procesamiento, administración y visualización gráfica de los datos, las redes TTN pueden sincronizar las aplicaciones para que se integren a plataformas en la nube como Evrythng IoT, myDevices Cayenne y OpenSensors.io. El propósito es que los datos transferidos sean útiles y accesibles.

\section{NODO FINAL PARA LA MEDICIÓN DE LA CALIDAD DE LA ENERGÍA ELÉCTRICA}

\subsection{OBJETIVO DE DISEÑO}

Uno de los objetivos es desarrollar un nodo final para una red de medición de la calidad de energía eléctrica con tecnología LoRaWAN, que cumpla con las características y especificaciones técnicas solicitadas, utilizando dispositivos y componentes electrónicos de última generación, para su uso en servicios de auditorías de calidad en pequeñas y medianas industrias.

\subsection{DIAGRAMA A BLOQUES DEL NODO FINAL}

Como primer paso del diseño, se elabora el diagrama a bloques de la tarjeta electrónica que define el nodo final de la red LoRaWAN (figura 2). 
Figura 2. Diagrama de bloques del nodo final.

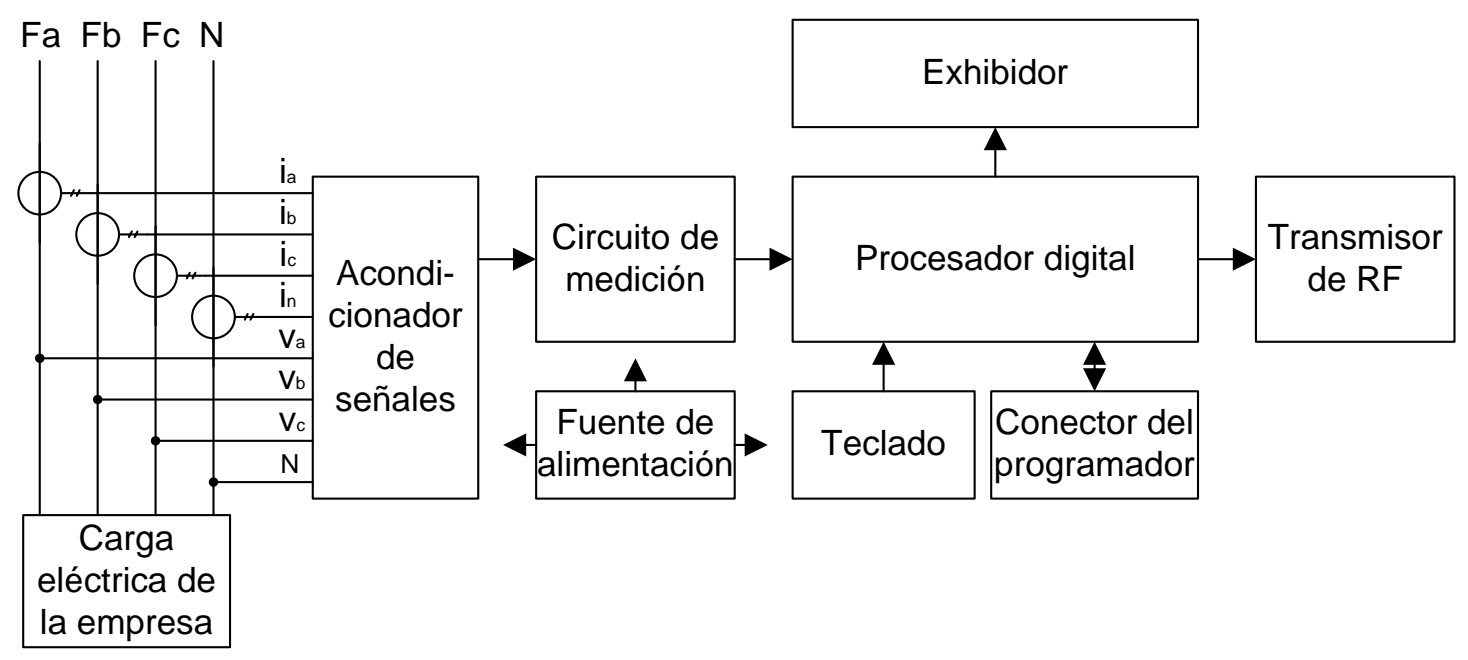

Los elementos principales de la tarjeta son el acondicionador de señales de corrientes y tensiones de la red eléctrica, la fuente de alimentación de $+3.3 \mathrm{~V}$ con respaldo de batería, el circuito de medición de variables eléctricas, el procesador digital y el módulo LoRaWAN transmisor de radiofrecuencia. Como elementos de interfaz se tienen el teclado y el exhibidor y como elemento de apoyo, el conector de programación.

\subsection{MÓDULO DE COMUNICACIONES}

Para la selección del módulo de comunicaciones de tecnología LoRaWAN, se hace un estudio de sus características de operación en la banda ISM de 915 MHz. Son dispositivos disponibles en el mercado, adecuados para la aplicación descrita (tabla 1).

Los módulos listados en la tabla 1 presentan características similares en cuanto a potencia de salida, sensibilidad, cobertura urbana, voltaje de alimentación y corrientes de transmisión y recepción. Las diferencias se presentan en la inclusión del protocolo LoraWAN, la interfaz a la unidad microcontroladora (MCU, por sus siglas en ingles), el precio, la disponibilidad en el mercado nacional y el fabricante. 
Tabla 1. Módulos de comunicación LoRaWAN propuestos

\begin{tabular}{|c|c|c|c|c|c|c|c|}
\hline $\begin{array}{c}\text { Parte / } \\
\text { fabricante }\end{array}$ & $\begin{array}{c}\text { Potencia } \\
\text { max. de } \\
\text { salida } \\
(\mathrm{dBm})\end{array}$ & $\begin{array}{c}\text { Sensibilida } \\
\text { d } \\
(\mathrm{dBm})\end{array}$ & $\begin{array}{c}\text { Cobertur } \\
\text { a } \\
\text { urbana } \\
(\mathbf{k m})\end{array}$ & $\begin{array}{c}\text { Protocol } \\
\text { o } \\
\text { LoRaW } \\
\text { AN }\end{array}$ & $\begin{array}{c}\text { Interfa } \\
z \\
\text { a MCU }\end{array}$ & $\begin{array}{c}\operatorname{Valim}(\mathbf{V}) / \\
\operatorname{Imax} \mathbf{T x} \\
(\mathbf{m A}) / \\
\operatorname{Imax} \mathbf{R x} \\
(\mathbf{m A}) \\
\end{array}$ & $\begin{array}{l}\text { Precio** } \\
\text { (USD) }\end{array}$ \\
\hline $\begin{array}{c}\text { EMB- } \\
\text { LR1272E / } \\
\text { Embit }\end{array}$ & +18 & -137 & 5 & $\begin{array}{c}\text { Clase A y } \\
\text { B }\end{array}$ & $\begin{array}{c}\text { UART, } \\
\text { I2C, } \\
\text { SPI }\end{array}$ & $3.3 / 125 / 12$ & $\begin{array}{l}30 \text { (solo } \\
\text { en USA) }\end{array}$ \\
\hline $\begin{array}{l}\text { Adafruit / } \\
\text { RFM95W }\end{array}$ & +20 & -139 & 5 & $\begin{array}{c}\mathrm{Si} \\
\text { incluido }\end{array}$ & SPI & $3.3 / 120 / 11$ & 25.94 \\
\hline $\begin{array}{l}\text { RN2903A / } \\
\text { Microchip }\end{array}$ & +18.5 & -146 & 5 & Clase A & UART & $\begin{array}{c}3.3 / 124 / \\
13.5 \\
\end{array}$ & 17.15 \\
\hline $\begin{array}{l}\text { inAir9B / } \\
\text { Modtronix }\end{array}$ & +20 & -148 & 5 & $\begin{array}{c}\text { No } \\
\text { incluido }\end{array}$ & SPI & $3.3 / 120 / 9.9$ & $\begin{array}{c}14.95 \\
\text { (solo en } \\
\text { USA) }\end{array}$ \\
\hline LoPy / PyCom & +20 & -137 & 5 & $\begin{array}{c}\text { Clase A y } \\
\text { C }\end{array}$ & $\begin{array}{c}\text { UART, } \\
\text { I2C, } \\
\text { SPI }\end{array}$ & $3.3 / 125 / 15$ & 28.69 \\
\hline
\end{tabular}

*Precios unitarios en México + IVA, al 30 de septiembre del 2018, si no se especifica otra cosa.

De acuerdo al prestigio del fabricante, precio y disponibilidad en el mercado, se selecciona el módulo RN2903A de Microchip. Aun cuando incluye el protocolo LoRaWAN solo para nodos clase A, esto es suficiente para la aplicación de medición de la calidad de la energía. De la misma manera, la interfaz UART es adecuada para una comunicación eficaz con el MCU, ya que se utiliza un conjunto simple de comandos ASCII, por lo que no representa una limitante.

\subsection{CIRCUITO DE MEDICIÓN DE LA CALIDAD DE LA ENERGÍA}

Las características básicas que se necesitan en el bloque de medición son que sea un circuito tipo AFE (del inglés Analog Front End) para redes eléctricas trifásicas, que incluya completamente las etapas de adquisición y procesamiento sin contener un MCU, que utilice transformadores o bobinas como sensores de corriente, además de tener una alimentación de $+3.3 \mathrm{~V}$, para mantener la compatibilidad con el módulo de comunicaciones. Para la medición de la calidad de energía, las características deseadas son:

* Medición de armónicas y distorsión armónica total de las variables eléctricas.

* Detección de sobretensiones y sobrecorrientes.

* Detección de la reducción y aumento de las tensiones de alimentación (tensiones sag y swell).

* Detección de tensiones y corrientes pico.

* Medición del periodo de las tensiones de alimentación.

* Detección de error en la secuencia de fases.

* Medición del factor de potencia.

De acuerdo a la tabla 2, se consideran dos fabricantes de circuitos de medición de la calidad de la energía eléctrica: Analog Devices y Microchip-Atmel. Analog Devices, empresa de amplia trayectoria en circuitos AFE de medición de variables eléctricas, presenta circuitos de última generación (ADE9000) y 
dispositivos consolidados en el mercado de los medidores de energía eléctrica (ADE7880). MicrochipAtmel, un nuevo competidor tecnológico en el ramo, ofrece el componente ATM90E36A, que de acuerdo a su hoja de datos, posee características similares al ADE9000, con la ventaja de su bajo costo.

Bajo este perfil, si se quiere un circuito que cumpla con las características y especificaciones deseadas al menor costo, la selección recae en el ATM90E36A; si la condición es tener la funcionalidad de monitoreo de la calidad de la energía, sin exceder en costo y consumo, la elección es el nuevo circuito de Analog Devices ADE9000. Si el interés es utilizar un circuito consolidado en cuanto a marca y operación, que cumpla con las características deseadas y que se encuentre en rangos aceptables de costo y consumo, la selección es el ADE7880. Ya que en este proyecto se quiere mantener la continuidad con trabajos anteriores y sobre todo con rutinas de software ya desarrolladas, se selecciona el ADE7880. No es el más económico, ni el que ofrece mejores prestaciones técnicas, pero si cumple con la funcionalidad y las condiciones deseadas.

Tabla 2. Circuitos AFE de medición de la calidad de la energía considerados.

\begin{tabular}{|c|c|c|c|c|c|c|c|}
\hline $\begin{array}{c}\text { Parte/ } \\
\text { fabricante }\end{array}$ & Mediciones & $\begin{array}{l}\text { Sensores de } \\
\text { corriente }\end{array}$ & Interfaz & $\begin{array}{l}\text { Exactitud } \\
\text { energía } \\
\text { activa } \\
(\%)\end{array}$ & $\begin{array}{c}\text { Otras } \\
\text { caracte- } \\
\text { rísticas }\end{array}$ & $\begin{array}{l}\text { Valim } \\
(\mathbf{V}) / \\
\operatorname{Imax} \\
(\mathbf{m A})\end{array}$ & $\begin{array}{l}\text { Precio* } \\
\text { (USD) }\end{array}$ \\
\hline $\begin{array}{l}\text { ADE9000 / } \\
\text { Analog } \\
\text { Devices }\end{array}$ & $\begin{array}{l}\text { 1/2,10/12 ciclos } \\
\text { RMS, activa, } \\
\text { reactiva y } \\
\text { aparente fund., } \\
\text { potencias } \\
\text { activa y } \\
\text { reactiva total, } \\
\text { distorsión } \\
\text { armón. total, } \\
\text { Irms, Vrms }\end{array}$ & $\begin{array}{l}\text { Transformador } \\
\text { de corriente, } \\
\text { Bobina } \\
\text { Rogowsky }\end{array}$ & $\begin{array}{l}\text { Pulsos, } \\
\text { SPI }\end{array}$ & 0.1 & $\begin{array}{l}\text { Monitor de } \\
\text { calidad de la } \\
\text { energía, } \\
\text { bajo } \\
\text { consumo, } \\
\text { Buffer de } \\
\text { formas de } \\
\text { onda, } \\
\text { encapsulado } \\
\text { LFCSP-40 P }\end{array}$ & $\begin{array}{ll}2.97 & \mathrm{a} \\
3.63 \quad / \\
17\end{array}$ & $\$ 17.45$ \\
\hline $\begin{array}{l}\text { ADE7880 / } \\
\text { Analog } \\
\text { Devices }\end{array}$ & $\begin{array}{l}\text { Pot. aparente, } \\
\text { activa y } \\
\text { reactiva } \\
\text { fundamentales, } \\
\text { pot. activa } \\
\text { total, distorsión } \\
\text { arm. total, } \\
\text { Irms, Vrms }\end{array}$ & $\begin{array}{l}\text { Transformador } \\
\text { de corriente, } \\
\text { Bobina } \\
\text { Rogowsky }\end{array}$ & $\begin{array}{l}\text { Pulsos, } \\
\text { I2C w/ } \\
\text { HSDC, } \\
\text { SPI }\end{array}$ & 0.1 & $\begin{array}{l}\text { Monitoreo } \\
\text { armónicas, } \\
\text { bajo } \\
\text { consumo, } \\
\text { muestreo de } \\
\text { formas de } \\
\text { onda, } \\
\text { encap. } \\
\text { LFCSP-40 P }\end{array}$ & $\begin{array}{ll}2.4 & \mathrm{a} \\
3.7 & / \\
25 & \end{array}$ & $\$ 18.54$ \\
\hline $\begin{array}{l}\text { ATM90E36A/ } \\
\text { Microchip- } \\
\text { Atmel }\end{array}$ & $\begin{array}{l}\text { Pot. activa, } \\
\text { reactiva } \\
\text { aparente, } \\
\text { potencia } \\
\text { fundamental y } \\
\text { arm., energías } \\
\text { activa, reactiva } \\
\text { y apar., } \\
\text { distorsión arm. } \\
\text { total, Irms, } \\
\text { Vrms }\end{array}$ & $\begin{array}{l}\text { Transformador } \\
\text { de corriente, } \\
\text { bobina } \\
\text { Rogowsky }\end{array}$ & $\begin{array}{l}\text { Pulsos, } \\
\text { SPI }\end{array}$ & 0.1 & $\begin{array}{l}\text { Bajo } \\
\text { consumo de } \\
\text { energía, } \\
\text { Detección de } \\
\text { eventos, } \\
\text { encapsulado } \\
\text { TQFP48 }\end{array}$ & $\begin{array}{ll}2.8 & \mathrm{a} \\
3.6 & / \\
23 & \end{array}$ & $\$ 4.21$ \\
\hline
\end{tabular}

*Precios unitarios en México + IVA, al 30 de septiembre del 2018. 


\subsection{DISPOSITIVO DE CONTROL Y PROCESAMIENTO}

El dispositivo de procesamiento que cumple con las funciones de control y procesamiento del nodo final es un microcontrolador (MCU). El segmento de los microcontroladores es uno de los de mayor crecimiento en la industria de semiconductores, ya que áreas tecnológicas como IoT, instrumentación industrial y electrónica automotriz, utilizan intensamente estos dispositivos. Esto permite que existan gran cantidad de fabricantes, sobresaliendo las siguientes empresas de acuerdo al ranking de ventas mundiales en el 2016: NXP (19\%), Renesas (16\%), Microchip (14\%), Samsung (12\%), ST (10\%), Infineon (7\%), Texas Instruments (6\%) y Cypress (4\%) (McGrath, 2017). Todas compiten ofreciendo productos de alto nivel y calidad a buen precio, por lo que seleccionar una u otra en función del costo, disponibilidad y funcionalidad de sus productos no es significativo. La elección de un MCU para grandes volúmenes de compra depende de las ventajas comerciales que ofrece el fabricante; las compras al menudeos dependens de las preferencias y experiencia del desarrollador, así como de las herramientas disponibles, ya que si no se tiene el soporte del simulador, el compilador $\mathrm{C}$ y el programador, aun cuando se tenga el mejor dispositivo, poco se lograría.

Tomando en cuenta que el módulo de comunicación LoRaWAN seleccionado es de la marca Microchip, se considera que una buena opción es utilizar uno de sus MCUs. Este fabricante ofrece cientos de microcontroladores, clasificados en función de la arquitectura interna, tamaño de la memoria de programa, tamaño de la memoria de datos, número de pines de entrada/salida, frecuencia máxima del CPU, número de puertos UART, SPI e I2C, entre otros parámetros.

Como referencia, el módulo LoRaWAN RN2903A utiliza internamente el MCU PIC18LF46K22 (Microchip, 2017), controlando un circuito transreceptor LoRa SX1276 de Semtech y alojando en su memoria el stack del protocolo LoRaWAN. Además, la información sugiere que el usuario puede incluir su programa de aplicación dentro del mismo chip, por lo que optar por este dispositivo es una opción aceptable.

En cuanto a la programación, se utiliza un sistema operativo en tiempo real cooperativo, para aprovechar al máximo las capacidades de los dispositivos electrónicos, principalmente del circuito ADE7880 y del módulo RN2903A.

\subsection{DISEÑO DE LOS OTROS BLOQUES}

Para el bloque acondicionador de señales de tensión y corriente, se considera el soporte que ofrece Analog Devices para el circuito ADE7880 (hoja de datos, diseños de referencia, foro de diseño, etc.). El diseño de la fuente de alimentación se basó en la revisión B del nodo final LoRa Mote de Microchip (Microchip, 2015-2016). Los bloques de teclado, conector de programación y exhibidor, son elementos 
típicos que se conectan a terminales de puertos paralelos de un MCU. En la figura 3 se observa una imagen de la tarjeta resultante.

Figura 3. Nodo final LoRaWAN de medición de la calidad de la energía eléctrica.

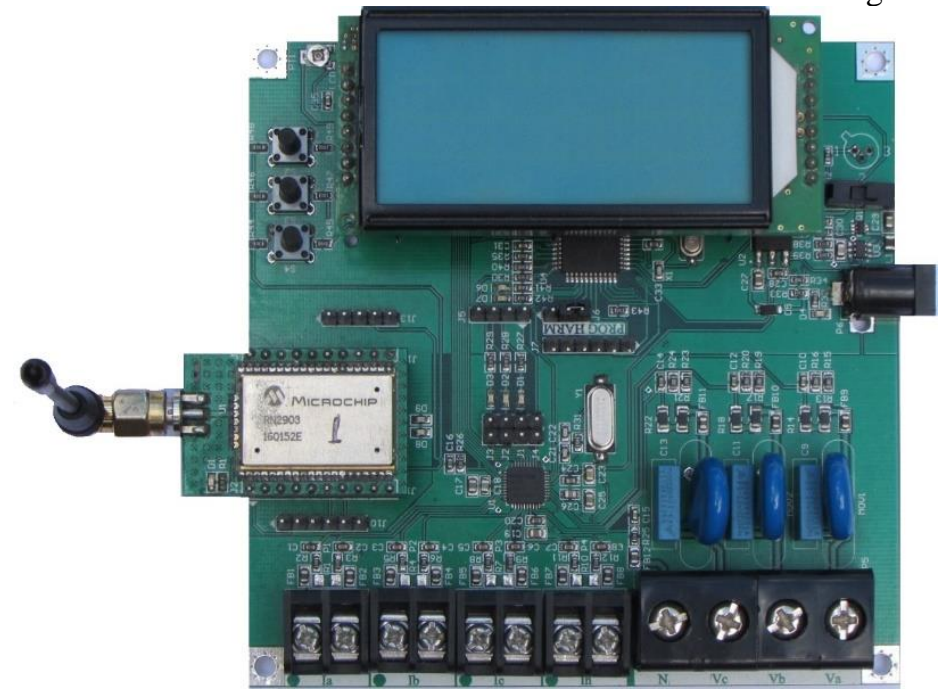

\section{COMENTARIOS FINALES}

Se ha realizado el diseño de un prototipo de nodo final para una red de IoT, con funciones de medición de la calidad de la energía en sistemas trifásicos. La tarjeta electrónica fabricada se conecta a una red LoRaWAN con el soporte de la organización TTN, para el acceso a los servidores de gateways, red y aplicaciones.

En un siguiente artículo se documentará la activación en la red del nodo final, la instalación de gateways internos y externos, el soporte para el manejo de datos de la organización TTN y el servicio de integración de la aplicación en la nube que se utiliza para el almacenamiento, procesamiento y toma de decisiones con los datos obtenidos. 


\section{REFERENCIAS}

Antonesei G. "Adaptive Real-Time DSP Architecture to Monitor Harmonic Components and Various Power Quality Factors in Electric Power Grids,” Analog Devices, Inc., Technical Article MS-2250, USA 2011.

Chandrappa, N. y S. Banerjee. "The next generation of power quality monitoring technology—Helping industrial equipment stay healthy,” Analog Devices, Inc., Technical Article, USA, 2017.

Components, RS. "11 Internet of Things (IoT) Protocols You Need to Know About," Design spark, April 20 2015, consultada por Internet el 29 de septiembre del 2018. Dir. de Internet: https://www.rsonline.com/designspark/eleven-internet-of-things-iot-protocols-you-need-to-know-about.

LoRa, A. "What is the LoRaWANTM Specification?," LoRa Alliance, consultada por Internet el 29 de septiembre del 2018. Dirección de Internet: https://lora-alliance.org/about-lorawan.

LoRaWAN. "LoRaWANTM Specification v1.1," LoRa Alliance, 2017, consultada por Internet el 29 de septiembre del 2018. Dirección de Internet: https://lora-alliance.org/resource-hub/lorawantmspecification-v11.

McGrath, D. "Qualcomm Poised to Become MCU Sales Leader?," EE Times 05.09.17, consultada por Internet el 30 de septiembre del 2018. Dirección de Internet: https://www.eetimes.com/author.asp?section_id=36\&doc_id=1331721.

Microchip, T. I. “LoRa® Mote User’s Guide,” Microchip Technology Incorporated, DS40001808B, 2015-2016, consultada por Internet el 30 de septiembre del 2018. Dirección de Internet: http://www.microchip.com/sitesearch/search/All/DS40001808B.

Microchip, T. I. "LoRaWANTM Library Plug-in for MPLAB® Code Configurator User's Guide," Microchip Technology Incorporated, DS40001864B, 2017, consultada por Internet el 30 de septiembre del 2018. Dirección de Internet: http://www.microchip.com/sitesearch/search /All/DS40001864B.

Quinnell, R. "Low power wide-area networking alternatives for the IoT," EDN Network, September 15, 2015, consultada por Internet el 29 de sept. del 2018. Dir. de Internet: https://www.edn.com/design/systems-design/4440343/Low-power-wide-area-networking-alternativesfor-the-IoT. 